\begin{tabular}{lc}
\hline & Journal of \\
Journal of Atmospheric and Solar-Terrestrial Physics 67 (2005) $1779-1785$ & ATMOSPHERIC AND \\
SOLAR-TERRESTRIAL \\
PHYSICS \\
\hline \hline www.elsevier.com/locate/jastp
\end{tabular}

\title{
The periodic spatial-temporal characteristics variations of the total ozone content
}

\author{
R.A. Sych ${ }^{\mathrm{a}, \mathrm{b}, *}$, G.K. Matafonov ${ }^{\mathrm{b}}$, A.Ju. Belinskaya ${ }^{\mathrm{b}}$, N.J. Ferreira ${ }^{\mathrm{a}}$ \\ ${ }^{a}$ National Institute of Space Research, Sao Jose dos Campos, 12.227-010 Brazil \\ ${ }^{\mathrm{b}}$ Institute of Solar-Terrestrial Physics, 664033, PO Box 4026, Irkutsk, Russia
}

Available online 26 August 2005

\begin{abstract}
This paper deals with quasi-periodical processes related to variations in the total ozone content (TOC) and solar radio emission at $10.7 \mathrm{~cm}$. For the detection of the hidden period in TOC variations, the wavelet transform was used to analyze the dynamic spectral analysis, the power spectra, and also the degree of correlations for selected harmonics. The harmonics obtained are related both to TOC variations and radio emission from the Sun, and they can be separated into four groups: (a) solar cyclic harmonics (periods of 5, 7, 11 and 22 years), (b) quasi-biennale modulation (about 2.5 years), (c) long periodical modulation with periods of 30, 60, 180 and 365 days, and (d) high-frequency modulation with a period of 6 days for ozone and 27 days for radio emissions. It is shown that the quasi-biennale harmonic represents a superposition of two close harmonics with periods of 1.8 and 2.7 years, the power maximum of which occurs in the minimum of solar activity. For the 6-day component, the expressed response is associated both with volcanic activities, having global character (e.g. eruption of the volcano Pinatubo), and solar flares with a noticeable proton component on July 13, 1982. It is also shown that there is a 180-day modulation of the climatic harmonic. Considering the zonal dependence of the integrated ozone power spectra, the latitude of $10^{\circ} \mathrm{S}$ was selected as the minimal power of the significance harmonics of the observed spectrum. The latitude asymmetry of the spectral component power change was also diagnosed.
\end{abstract}

(C) 2005 Elsevier Ltd. All rights reserved.

Keywords: Quasi-periodical processes; Wavelet analysis; Total ozone content; Radio emission

\section{Introduction}

The problem of the possible influence of solar activity variations on atmospheric, climate and weather processes has attracted researchers' attention for years. It is known that the ozonosphere, as a part of the terrestrial atmosphere, plays a key role in many processes, determining global climate changes and connections with various atmospheric layers.

\footnotetext{
*Corresponding author. Institute of Solar-Terrestrial Physics, 664033, PO Box 4026, Irkutsk, Russia. Fax: +7 (3952) 425557.

E-mail address: sych@iszf.irk.ru (R.A. Sych).
}

The first results concerning the influence of solar activity on changes of total ozone content (TOC) were considered by Paetzold studies (Paetzold, 1969; Paetzold et al., 1972). The analysis of vertical sounding using Paetzold-Kulke optical ozone probes for the 1958-1968 period has shown that there is a dependence between TOC changes and the phase of solar activity. Analysis of the reasons that would support this finding (including changes in the circulation winds, and also the photochemical reactions resulting in the ozone formation) could not explain the results observed. Therefore, a hypothesis concerning the change of an ultra-violet component of solar emission with a phase of solar activity was assumed and an 11-year modulation of 
formation of ozone in the atmosphere was explained. Further observations obtained in the work of Angell and Korschover (1978) have confirmed increases in the ozone contents at heights above $16 \mathrm{~km}$ from the minimum to the maximum of a solar cycle. It was also shown that emission at 160-210 nm wavelength changes more than $60 \%$ depending on the intensity of calcium floccules (Prag and Morse, 1970). Once more, a component of solar emission is the factor in a relative number of solar spots. The analysis of stratosphere measurements carried out by London and Dutsch (1977) has shown a correlation between TOC and the number of solar spots. Comparison of emissions close to the $122 \mathrm{~nm}$ spectral line and TOC (Nimbus-4) for 10 days (Blackschear and Tolson, 1978) was also done. In all cases, the data on TOC variations and solar factors showed a good correlation.

Another hypothesis on the TOC variation was the assumption that there is a solar modulation of a nitric oxide source, created by galactic rays at high latitudes in the stratosphere (Ruderman and Chamberlain, 1975). The further calculation of the Forbush effect caused such modulation to show that this contribution is minimal (Crutzen, 1975).

A special role in the short-term change of the TOC is played by powerful X-ray flares. The first assumptions about the influence of high-energy particles (protons) during a solar flare were proposed by Zerefos and Crutzen (1975). A similar study was executed for a proton flare on July 131982 (Solomon et al., 1983).

It is important to mention that all previous studies searching for a correlation between solar activity and the TOC were carried out for signals containing total modulated components with different weight factors, which can describe relationships only in general. Therefore, it is very important to understand the contribution of each spectral component in integrated signals (e.g. TOC), radio emission and their relationships.

In this paper, we present a new wavelet-based strategy for obtaining spectral characteristics of frequency harmonics and their correlation between sets of the TOC and solar radio emission at $10.7 \mathrm{~cm}$. Experimentally, we tested the reliability of a 6-day component response in association with volcanic activities on a global scale and the importance of solar flares (proton component) for change in the TOC. For fluctuations in the TOC, significant periods are obtained and a correlation was shown between them with similar periods in radio emission $(10.7 \mathrm{~cm})$ from the Sun.

\section{Instrumentation}

The dataset used in this study comprises observation of the TOC, measured by the total ozone mapping spectrometer (TOMS) on board the Nimbus-7 satellite during the 1979-1992 period. The data are daily averages on a spatial grid with a $1^{\circ} \times 1.25^{\circ}$ (latitudelongitude) cell. The TOC (in Dobson units) is defined as the absorption of diffused solar ultra-violet emission at different wavelengths (312 and $331 \mathrm{~nm}$ ); the measurement error is $\sim 2-4 \%$. Innumerous comparisons of these data with ground measurement devices have already shown their reliability. NASA distributes TOC data on compact discs in various package versions. In the current study, the time series of the TOC with daily temporal resolution on a spatial grid with a $5^{\circ}$ latitude and longitude resolution were investigated. Also, the solar activity impact on TOC changes was found using the Sun radio emission data at $10.7 \mathrm{~cm}$, received by the solar radio monitoring from the Dominion Astrophysical Observatory (Canada). The accessible database includes two components, the measurements of the Sun radio flux during the day and its average value. Each radio flux can be presented in three aspects: "observable", "corrected" and the so-called "URSI Series D". The "observable" events are the direct response by the antenna system on the received radiation. It is modulated by two components: the level of solar activity and the change in Earth-Sun distance. The "corrected" ones are the flux where the annual change was corrected and the flux for the average distance between Sun-Earth is given. In the case of the "URSI Series D" data, to apply absolute binding of a radio flux to density flux on different frequencies (spectral characteristics), the scale factors were used. In our case we used the "observable" radio flux to study events connected with the terrestrial and space factors. The flux of radio emission at $10.7 \mathrm{~cm}$ is given in terms of solar flux unit (SFU, $1 \mathrm{SFU}=10^{-22} \mathrm{~W} \mathrm{~m}^{-2} \mathrm{~Hz}^{-1}$ ).

\section{Experimental data}

Fig. 1a shows the time dependence of the daily solar radio emission for the $01 / 01 / 1979-12 / 31 / 1992$ period; the dataset length is 5112 days. In this figure, the global flux modulation is clearly seen as two 11-year solar cycles (21 and 22) with an increase in the power of highfrequency components in a growth phase and during the maximum activity of intensive short-term emissions. Similar behavior is typical for changes in solar activity and connected to a 27-day time period rotation of the solar surface. This feature is well traced in the example of the occurrence and disappearance of solar group spots generating radio emission at $10.7 \mathrm{~cm}$.

To study the solar activity influence on the TOC, we used coincident time series of the latter variable and radio-emission data. The equatorial flux variation was analyzed from $0^{\circ}$ to $360^{\circ}$ of longitude every $5^{\circ}$, and for a fixed $120^{\circ}$ longitude band from $60^{\circ} \mathrm{N}$ to $60^{\circ} \mathrm{S}$. An example of TOC variation as a function of time for an 

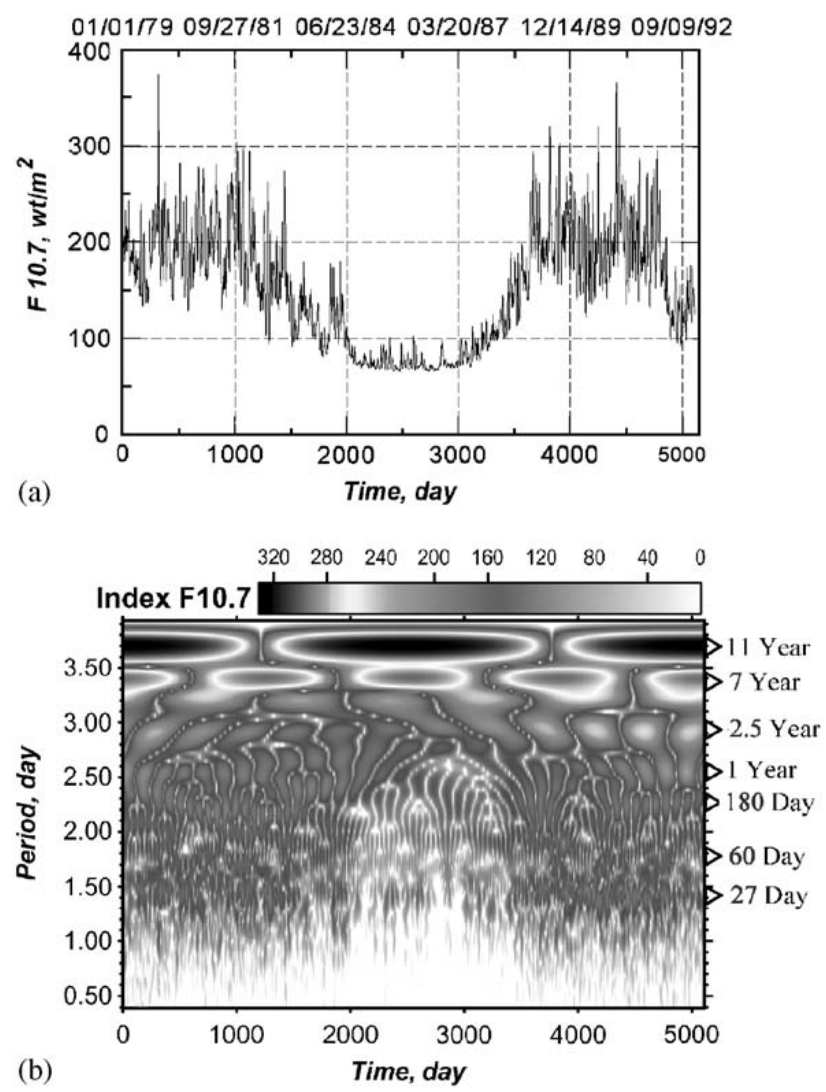

Fig. 1. (a) Time profile of radio emission $(10.7 \mathrm{~cm})$ obtained on Dominion Radio Astronomy Observatory (Canada). The time cadence is 1 day, period observation from 01.01.1979 to 31.12.1992. (b) Amplitude wavelet spectra of time series with indication of significant periods (27, 60 and 180 days; $1,2.5,7$ and 11 years). Intensity of wavelet coefficients and periods are presented in logarithmic scale.

equatorial area along $120^{\circ} \mathrm{E}$ is shown in Fig. 2a. Intensive quasi-periodical modulation is well visible both in TOC as well as in $10.7 \mathrm{~cm}$ flux variations.

\section{Methodology}

To obtain hidden information, the built-in in quasiperiodical fluctuation, we applied a new mathematical apparatus, actively being developed and having many practical applications-1D wavelet analysis (Chui, 1992). It is known that any signal of a natural origin represents a set of spectral harmonics, which can be presented as the sum of regular harmonics only under specific approximation. Thus, the physical interpretation of such a signal is problematic. Using standard Fourier decomposition, we cannot consider interfering factors such as the evolution of a frequency signal over time, the change in power of separate harmonics over the time of the development process, and also influences from fast signal changes, called natural and human factors, which
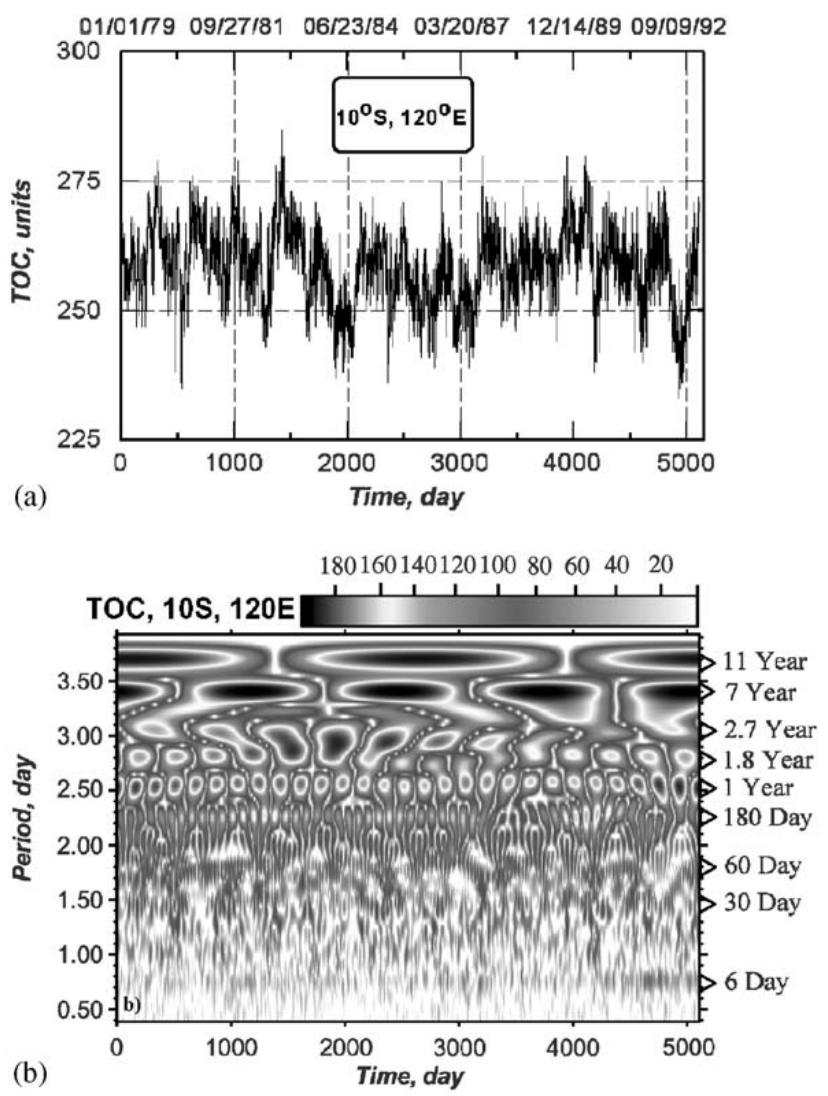

Fig. 2. (a) Time profile of total ozone content (TOC) during 01.01.1979-31.12.1992. Data obtained by TOMS (Total Ozone Mapping Spectrometer) sensor on board the Nimbus-7 satellite for near-equatorial zone $10^{\circ} \mathrm{S}, 120^{\circ} \mathrm{E}$. (b) Amplitude wavelet spectrum of the TOC time series. Arrow is specified significant periods of the spectrum $(6,30,60$ and 180 days; $1,1.8,2.7,7$ and 11 years). Intensity of wavelet coefficients and periods are presented in logarithmic scale.

bring errors to the power of harmonics in the integral spectrum. In the Fourier decomposition (Astaf'eva, 1996), an initial signal $f(t)$ is multiplied by $\mathrm{e}^{-\mathrm{i} \omega t}$ and integrated in time from $-\infty$ up to $+\infty$. If the signal does not contain spectral components with frequency $\omega$, the integration result will be zero. On the other hand, if a signal during any time interval is present in a spectral component with frequency $\omega$, the integration result of this component will be determined only by the duration of this time interval and will not depend on it. These difficulties are successfully overcome with the wavelet transform method.

The basis of the wavelet analysis is the convolution of a two-parametrical basic function with a signal by means of scale dilations and translation (Daubechies, 1988, 1990, 1991). As opposed to an indefinitely oscillating sinusoidal wave, which forms an orthonormalized basis in the Fourier transform functional space $L^{2}(0,2 \pi)$, the basic function in the wavelet transform is localized from determined spectral properties, with a fast drift to zero in a small time interval. The dilation 
and translation system, whose value is a multiple of two, is applied to cover both frequency and time spatial axes. The wavelet transform integral is written as

$\left[W_{\psi} f\right](a, b)=|a|^{-1 / 2} \int_{-\infty}^{\infty} f(t) \psi^{*}\left(\frac{t-b}{a}\right) \mathrm{d} t$,

where $a$ is the scaling coefficient, $b$ the translation parameter and the basis or "mother" function $\psi(t)$ is given by

$\psi_{a b}(t)=|a|^{-1 / 2} \psi\left(\frac{t-b}{a}\right), \quad a, b \in R, \quad \psi \in L^{2}(R)$.

The choice of analyzing the "mother" function is determined by the type of information necessary in order to obtain the studied signal. The basis for constructing various types of basic functions are the derivative Gauss functions. Each of these functions has its own name-MHAT (Farge, 1992), WAVE, Morlet (Grossmann and Morlet, 1984), LBM (Lemarie and Meyer, 1986), Paul (Paul, 1984) and behavior in frequency-temporal space. The combination of the frequency localization to an opportunity of temporary binding of separate non-stationary processes allows the revealing of such features of temporary sets as a change of frequency in time, the power change in separate harmonics, analyzing local regularities, and building both local and global power spectra. In the current study, a complex Morlet "mother" function, which has the optimum spectral resolution both in temporal and spatial scales, was applied.

The wavelet spectrum of a time series represents a function of two variables (frequency and time). Therefore a contour map was used for its graphic representation. This type of spectra visualization depicts the time changes of the amplitudes of wavelet coefficients and their frequency characteristics. The amplitude analysis both on high and low frequencies, having strong differences in amplitudes, was done on a logarithmic scale. The estimation of the reliability of the selected frequencies was done on the basis of the building of wavelet spectra on the noise components. For many geophysical events, such components are white noise, having a flat Fourier spectrum, or "red" noise, described by the increase of power with decreasing frequency.

\section{Result and discussion}

Fig. $2 b$ shows the amplitude wavelet spectrum of a TOC time series for a geographical location with coordinates $10^{\circ} \mathrm{S}$ and $120^{\circ} \mathrm{E}$. The coefficients of the wavelet transform are presented on a time-period axis with the indication of their amplitudes in a logarithmic scale.

Analyzing the amplitude of the wavelet spectrum, it is possible to note rather steady quasi-periodical processes, which are observed on the selected harmonics. The strongly marked annual component and also the 30,60 and 180th day harmonics are prominent. All these spectral components weakly vary in time and their amplitudes change in correlation with the basic harmonic (365 days). The next clearly observed component in the spectrum is a quasi-biennial cycle with a period around 2.5 years. Its power changes with time, reaching a maximum at the end of the 21 solar cycles. Thus, as opposed to the annual components with a constant period, the quasi-biennale component represents the sum of two close-range harmonics with periods of 1.8 and 2.7 years. They are clearly separate near the solar activity maximum and merge in the minimum, forming one harmonic component with a 2.5-year period. This component is probably connected to the well-known quasi-biennial fluctuations of the stratospheric wind above the equator (QBO-variation).

Moreover, the low-frequency components -5 and 7 years - can represent multiple components of the 11year cycle of solar activity because the influence of the Sun on the TOC is basically connected to the increasing power of the ultra-violet emission from active regions. It is possible to note on the spectrum that the visible and 22-year components characteristic of solar activity variations are also evident.

The 6th day component is clearly seen on an integral power spectrum. Considering temporal behavior on different longitude along the equator, it is possible to notice a burst-like character change in intensity (Fig. 4). The maxima of intensity are marked on September 8 1980, July 27 1982, August 281986 and August 25, 1991. Two bursts-in July 1982 and in August 1991 can be connected both with flares of solar protons and with strong volcanic emission (July 13 1982-protons, June 1991-volcano Pinatubo). Thus, if in the first case the influence has a local character, in the second one (volcanic activity) there is a global influence on the TOC. It is also necessary to note the 180th-day modulation of 6th-day components, clearly expressed as its summation on various longitudes.

Spectral processing, similar to the one described above, was also done for the $10.7 \mathrm{~cm}$ radio flux time series. The annual and semi-annual modulating components (Fig. 1b) are clearly allocated. The natural occurrence of these harmonics is connected to cyclic change of the Sun-Earth distance, whose modulation makes the basic contribution. It is seen that these components are absent in the minimum of solar activity, and at the stage of growth activity their occurrence is connected to splitting annual components into two components - 365 and 180 days. The quasi-biennial component disappears in a minimum of solar activity. The amplitudes of low-frequency components of a solar cycle -5 and 11 years - are constant with time. Similar to the 6th-day time component of the TOC dataset, a 27- 
day time component is also observed in the radio emission data, which is a good marker of occurrence and disappearance of active areas.

To evaluate the relation between TOC spectra and radio emission, a correlation analysis of the wavelet harmonics on Global Wavelet Spectra (GWS) was carried out (Fig. 3a). It is shown that the correlation coefficients increase with the period of existing harmonics and that for the global harmonics-1, 5, 7, 11 years they are close to 0.9 (Fig. 3b). There is some correlation of the 6th-day radio and TOC component, indicating a relationship between them. (Fig. 4). The change of the quasi-biennial components for the two datasets occurs in anti-phase (coefficient of correlation about -0.9 ). The anti-correlation of the amplitude variations of the TOC and radio-emission harmonics is also observed for the 180-day components.

The latitude and longitude variations of TOC power spectral components were also analyzed. With this
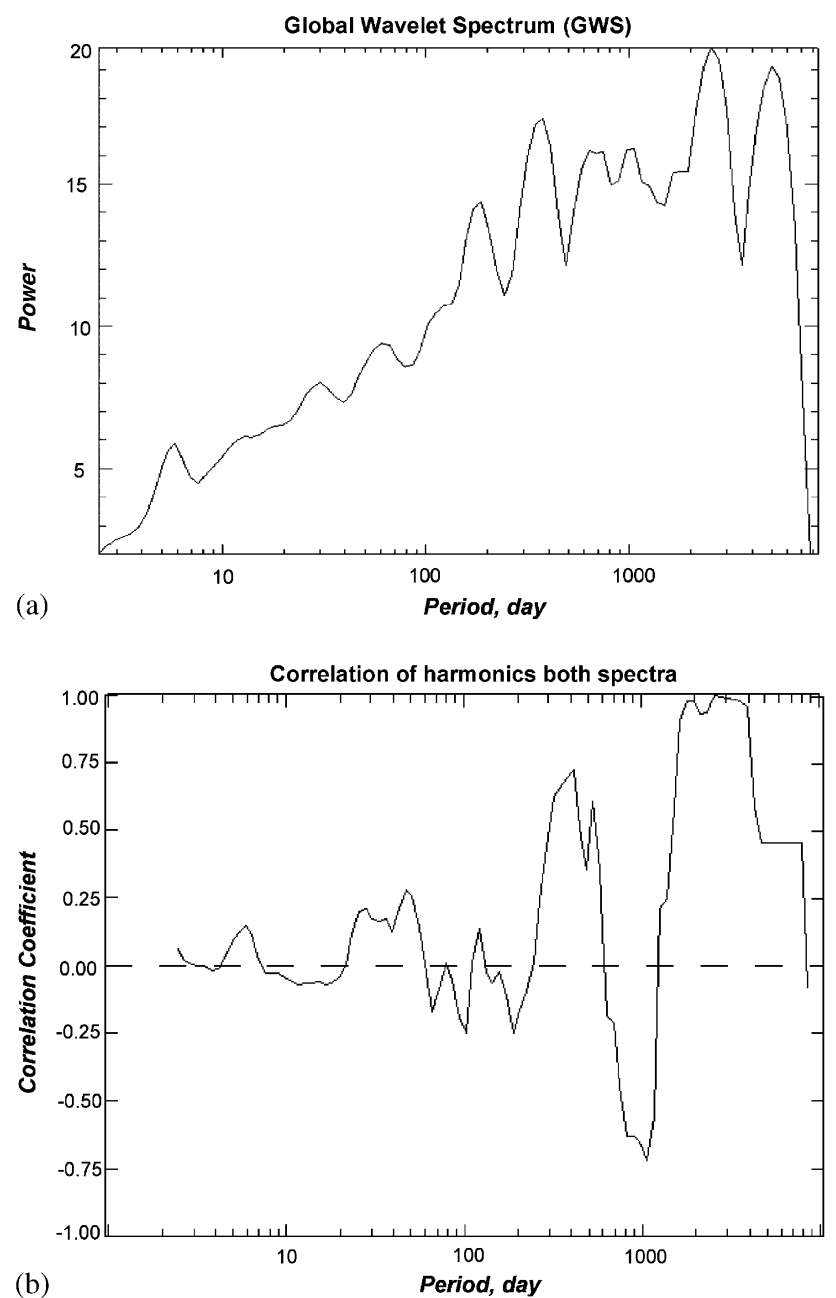

Fig. 3. (a) Global wavelet spectrum (GWT) of the TOC time series for points with coordinates $10^{\circ} \mathrm{S}, 120^{\circ} \mathrm{E}$. (b) Profile of mutual correlation between frequency harmonics of two wavelet spectra: radio emission and total ozone content.

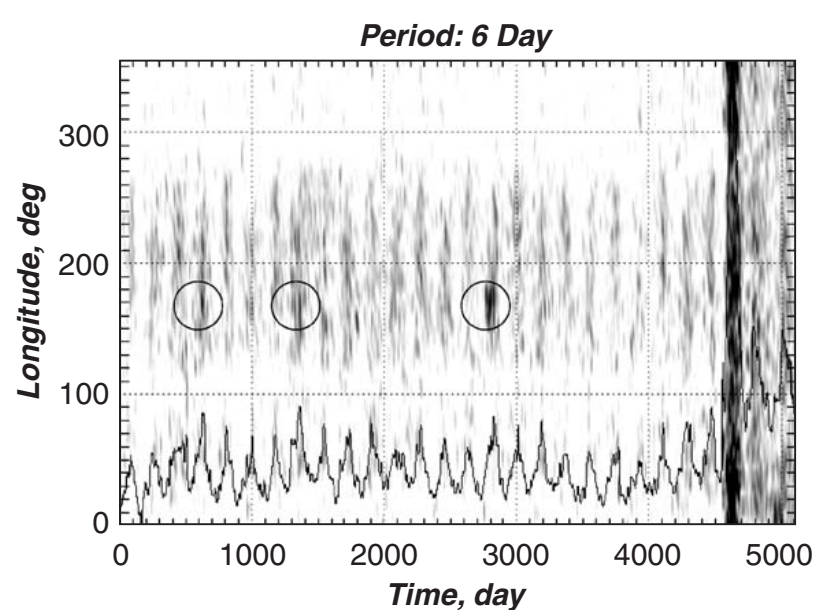

Fig. 4. Longitude-temporary behavior of the 6th-day harmonic of a total ozone content wavelet spectrum. The temporary variations of intensity connected with both proton component of solar flares (local influence, circles) and volcanic activity (global influence, volcano Pinatubo, dark strip) are shown. The integrated curve of variation 6th-day harmonic on all longitudes is superimposed on the image and shows 180th-day time modulation.

purpose, the wavelet analysis of TOC time series at various latitudes (from $60^{\circ} \mathrm{N}$ up to $60^{\circ} \mathrm{S}$ every $5^{\circ}$ ) along $120^{\circ} \mathrm{E}$ was carried out. A similar calculation was made along the equator in scattered points from $0^{\circ}$ up to $360^{\circ}$ every $5^{\circ}$ of longitude. The wavelet spectrum was also calculated for each point. It is known that the full energy of the wavelet spectrum is distributed according to the global distribution power of the coefficients in the wavelet transformation. At each moment of time it is possible to calculate a local spectrum of energy. The total level of energy in all time scales results in the socalled GWS. Compared with the usual Fourier spectrum, it has a smoother character at high frequencies and a good frequency resolution at low frequencies. This type of spectrum is defined by the frequency characteristics of the basic function. The changes of its characteristics can improve the temporary resolution and worsen the frequency, or vice versa.

Fig. 5a shows a contour map calculated from the GWS at various latitudes (meridian $120^{\circ} \mathrm{E}$ ). It is seen that there is a selected latitude $-10^{\circ} \mathrm{S}$, where the minimum power of all selected harmonics $(6,30,60$, 180 and 365 days, and also 2.5, 5, 7, 11 and 22 years) is located. The power of the harmonics is increased when moving from this zone and in the southern hemisphere, the rise exhibits a smooth character while in the northern hemisphere some plateau of power in the $10-35^{\circ} \mathrm{N}$ sector is observed. As shown in Fig. 5, marked earlier "mirrority" from an annual track TOC in northern and southern hemispheres (Reinsel et al., 1982, 1983), it takes place only in the first approximation and its behavior varies for different harmonics. It 

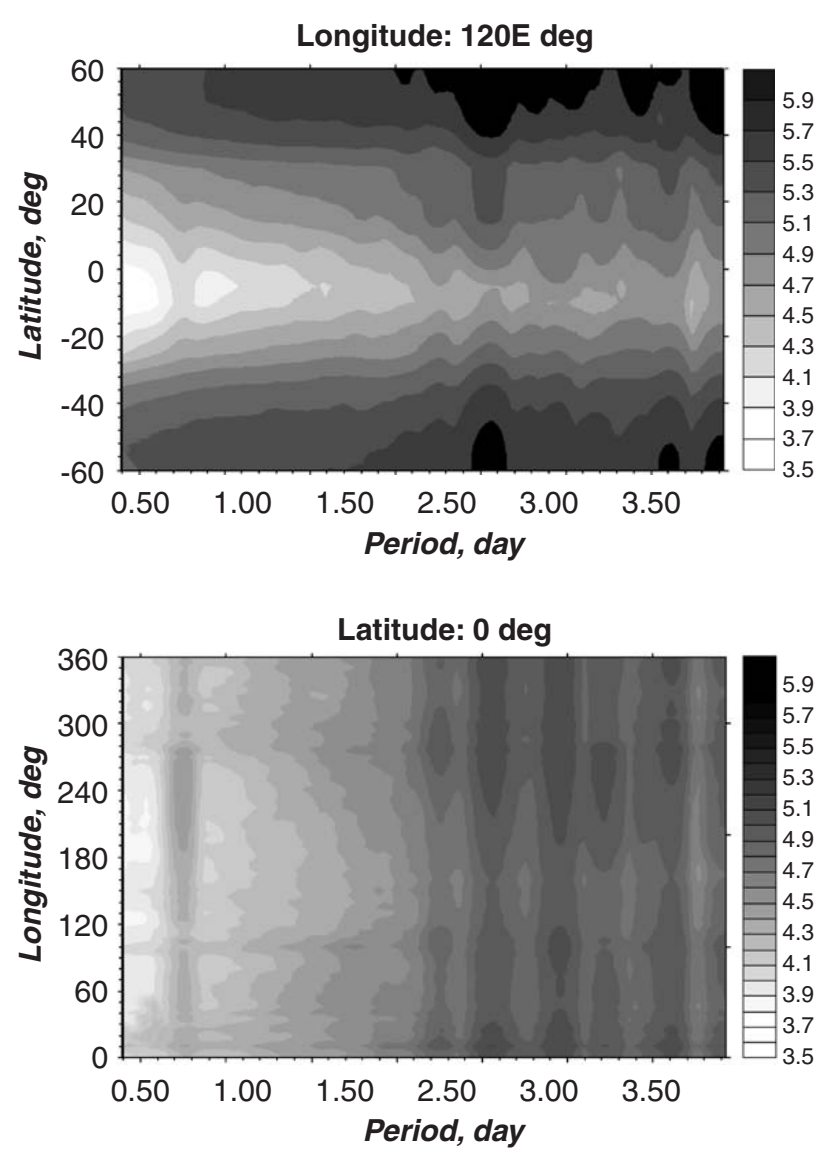

Fig. 5. Values of harmonics in global wavelet spectra of total ozone content, depending from latitudes (a, $120^{\circ} \mathrm{E}$ longitude) and from longitudes (b, equator). The power of harmonics is shown by various levels of gray intensity. The periods are presented in logarithmic scale.

should be noted that the harmonics near the equatorial zone are most contrasted and their power is minimum. The increasing of latitude leads to power growth in all signal spectrum components, but on the other hand, there is a decrease in number of significant harmonics and an increase in the high-frequency noise component.

In Fig. 5b, we can observe the behavior of the same components along the equator as a function of geographical longitudes. The power of the harmonics is distributed non-uniformly with longitude and with appreciable increase in separate regions.

\section{Conclusion}

In this study, we applied wavelet analysis for the TOC and radio emission $(10.7 \mathrm{~cm})$ time series to obtain information about the interaction of these two heliophysical factors on the basis of significant spectral harmonics. The obtained spectral decomposition has shown similar harmonics both related to TOC variations and radio emission of the Sun. They can be divided into harmonics associated with solar periodicity $(5,7,11$ and 22 years), quasi-biennale modulation ( 2.5 years), long periodical modulation (30,60, 180 and 365 days) and high-frequency modulation with periods of 6 and 27 days, respectively, for TOC and radio emission.

To analyze the longitude and latitude dependence of TOC variations, we have applied wavelet analysis to the time series of this variable for various geographical locations. It is shown that the minimum power of all the wavelet spectrum harmonics occurs at latitude $10^{\circ} \mathrm{S}$.

The behavior of 6-day components for various longitudes shows a global character-response to volcanic activity (eruption of Pinatubo) and a local characterthe solar flare of July 13, 1982. This feature occupies only a small-frequency part of the spectra, in the lowfrequency part essentially there is no change.

To obtain the correlation between two wavelet spectra (TOC and radio emission), we calculated the dependence between coefficient correlations of both harmonic spectra. We can see that there is a good correlation $(k=0.9)$ for the low-frequency component $(1,5,7$ and 11 years). Also, variation of the quasi-biennale components of the TOC occurs in anti-phase compared with the radio emission of the Sun.

The applicability and suitability of wavelet analysis to study the spatial-temporal variations of the TOCs was explored in this study. Further research should expand the sphere of its application to create the base for physical interpretation of the observed quasi-periodical structure.

\section{Acknowledgments}

The authors thank the National Space Science Data Center at NASA, USA for providing TOMS/NIMBUS7 ozone data. The work was supported by the Russian Foundation for Basic Research-Grant 477.2003.2, Grants NSFC 10225313 and FAPESP (2003/06665-2).

\section{References}

Angell, J.K., Korschover, J., 1978. Global analysis of recent total ozone fluctuations. Monthly Weather Review 106, 725.

Astaf'eva, N.M., 1996. Wavelet analysis: basic theory and some applications. Uspekhi Fizicheskikh Nauk 166 (N11), $1145-1170$

Blackschear, W.J., Tolson, R.K., 1978. High correlation between variations in monthly averages of solar activity and total atmospheric ozone. Geophysical Research Letters 5 (N 11), 921-924.

Chui, K. (Ed.), 1992. Wavelet Analysis and its Applications, vol. 1. An Introduction to Wavelets, vol. 2. Wavelets: A Tutorial in Theory and Applications. Academic Press Inc., New York. 
Crutzen, P.J., 1975. A two dimensional photochemical model of the atmosphere below $55 \mathrm{~km}$ : estimates of natural and man caused ozone perturbations due to NOx. Proceedings of the Fourth Conference of CIAP, pp. 264-279.

Daubechies, I., 1988. Communications in Pure and Applied Maths 41, 906.

Daubechies, I., 1990. IEEE Transactions on Information Theory 36, 961.

Daubechies, I., 1991. Ten Lectures on Wavelets. CBSM Lecture Notes Series. SIAM, Philadelphia.

Farge, M., 1992. Wavelet transforms and their applications to turbulence. Annual Review of Fluid Mechanics 24, 395-457.

Grossmann, A., Morlet, J., 1984. Decomposition of Hardy functions into square integrable wavelets of constant shape. SIAM Journal on Mathematical Analysis 15, 723-736.

Lemarie, P.G., Meyer, Y., 1986. Ondelettes et bases Hilbertiennes. Rev. Math. Ibero-Americana 2, 1.

London, J., Dutsch, H.V., 1977. Variations including possible solar cycle variations of stratospheric ozone over Central Switzerland. Proceedings of the Joint Symposium on Atmospheric Ozone, Berlin, vol. 1, pp. 289-302.

Paetzold, H.K., 1969. Variations of the vertical ozone profile over middle Europe from 1951 to 1968. Annales de Geophysique 25, 347-349.

Paetzold, H.K., Piscalar, F., Zschozner, H., 1972. Secular variation of the stratospheric ozone layer over middle Europe during the solar cycles from 1951 to 1972 . Natural Physical Science 240, 106-107.
Paul, T., 1984. Functions analytic on the half-plane as quantum mechanical states. Journal of Mathematical Physics 25 (11), 3252-3263.

Prag, P.S., Morse, J.A., 1970. Variations in the solar ultraviolet flux from July 13 to August 9, 1968. Journal of Geophysical Research 75 (N25), 4613-4621.

Reinsel, G., Tiao, G.C., Lewis, R., 1982. A statistical analysis of total ozone data from the Nimbus-4 BUV satellite experiment. Journal of Atmospheric Science 39 (N2), 418-430.

Reinsel, G., Tiao, G.C., Lewis, R., Bobkoski, M., 1983. Analysis of upper stratospheric ozone profile data from the ground-based Umkeyr method and the Nimbus-4 BUV satellite experiment. Journal of Geophysical Research C 88 (N9), 5393-5402.

Ruderman, M.A., Chamberlain, G.W., 1975. Origin of the sunspot modulation of ozone: its implications for stratospheric NO injection. Planetary and Space Science 23, 247-268.

Solomon, S., Reid, G.C., Rusch, D.W., Thomas, R.J., 1983. Mesospheric ozone depletion during the solar proton event of July 13, 1982. Part II. Comparison between theory and measurements. Geophysical Research Letters 10 (N4), 257-260.

Zerefos, C.S., Crutzen, P.J., 1975. Stratospheric thickness variations over the Northern Hemisphere and their possible relation to solar activity. Journal of Geophysical Research 80 (N 39), 5041-5043. 